\title{
Partial Discharge Source Classification and De-Noising in Rotating Machines Using Discrete Wavelet Transform and Directional Coupling Capacitor
}

\author{
Mohammad Amin Kashiha, Diman Zad Tootaghaj, Dolat Jamshidi \\ Communications Department, Niroo Research Institute (NRI), Tehran, Iran. \\ Email: makashiha@gmail.com,diman_zad@yahoo.com,djamshidi@nri.ac.ir
}

Received March $28^{\text {th }}, 2009$; revised May 22 ${ }^{\text {nd }}, 2009$; accepted May $28^{\text {th }}, 2009$.

\begin{abstract}
This paper introduces a new method to separate $P D^{1}$ from other disturbing signals present on the high voltage generators and motors. The method is based on combination of a pattern classifier, the Discrete Wavelet Transform (DWT), to de-noise PD and Time-Of-Arrival method to separate PD sources. Furthermore, it will be shown that it can recognize $P D$ sources including rotating machine's internal and external discharge pulses (e.g. on the bus bar).
\end{abstract}

Keywords: Partial Discharge, Discrete Wavelet Transform, Time-Of-Arrival, Rotating Machines, De-Noising, Coupling Capacitor

\section{Introduction}

As a result of deterioration of insulating systems in high voltage equipments, small electrical spikes occur within the insulation [1]. This could cause further degradation of insulation and finally failure of the equipment. So, insulation assessment of these equipments is necessary in order to avoid catastrophic consequences. During last decades a huge number of studies have been done on recognizing Partial Discharge (PD) pulses in high voltage equipments including rotating machines. Although there have been good achievements in this field [1], still there is a long way to introduce a method to separate PD from noise and interferences in a perfect way. There are several approaches to extract PD and there are a handful of papers on each of them. Reference [2] discusses a method based on fuzzy classification of PD. Although it is partially successful in recognizing cross-coupling resulted from adjacent phases, its authors admit that certainty of their method is not high. There are also some methods based on artificial intelligence [2] but they suffer from low generality and high calculation needs. Nowadays it is proved that time-frequency transforms such as Wavelet $[3,4]$ and Hilbert-Huang transform [5] have the best performance in PD de-noising. As authors investigated al-

${ }^{1}$ Partial Discharge most all of published methods and most of survey papers approve it too [6,7], the best and most successful method to extract PD from noise and interferences is DWT. Hence, the approach was developed based on DWT de-noising but the method suffers from disability of separating PD originating from rotating machine and the bus bar. Thus, a complementary technique (Time-Of-Arrival) which is based on the method introduced in [8] was used. The idea of the latter is based on directional coupling capacitors as conventional sensors used to attach measurement instruments to high voltage windings.

The paper is organized as follows: Section 2 clarifies problems dealt with in PD de-noising. Section 3 discusses DWT and its application in PD de-noising. Section 4 explains the Time-Of-Arrival technique. Section 5 discusses the results obtained and Section 6 concludes the paper.

\section{Problem Definition}

PD measurement is a main concern of operators dealing with generators and motors as they want to avoid machine failure. On the other hand, they prefer to do this while the machine is operating (i.e. on-line measurement) because detaching a generator from the network is costly and time-consuming. Meanwhile in on-line operation of 
these machines, there are different kinds of noise and interference signals that make measurements unreliable. Therefore, a method is needed to separate PD from these signals. The method this paper follows is based on DWT which is a time-frequency transform. As known, PD is a non-stationary signal [9]. So, conventional transforms such as Fourier Transform (FT) may not be used to analyze spectral specifications of PD as they do not distinguish short-term and long-term frequency components. But DWT considers time events of the signal. Thus, it is capable of interpreting short-time PD pulses. Next section discusses PD de-noising using DWT.

\section{PD De-Noising Using DWT}

Wavelets have very attractive features which cause them to be used in miscellaneous applications [10]. One of the methods which works based on these features is decomposing and reconstructing signals using $\mathrm{QMF}^{2}$ filters. Reference [2] is a good context to understand how DWT decomposition using QMF filters works. But here the focus is on applying this technique to develop the method. In general, DWT decomposes a signal to its basic frequency components as shown in Figure 1.

Recorded signal from sensors includes both PD and noise. It is known that noise has a stochastic nature. So, it is expected that its energy ${ }^{3}$ is divided equally between filter bands. But PD's energy is mostly concentrated in a few bands [9]. Energy of the coefficients is a reliable criterion to separate bands which may contain PD more than noise. Author's experience showed that using 2nd order Daubechies (db2) mother wavelet [9], more than $80 \%$ of PD signal's energy is gathered in one of the detail coefficients (cDs) of DWT. This is a useful result which could be considered to determine global threshold and finally separate PD from noise. Energy distribution of a sample is shown in Figure 2. As it is seen cD6 (detail coefficient of 6th decomposition level) includes most of PD's energy.

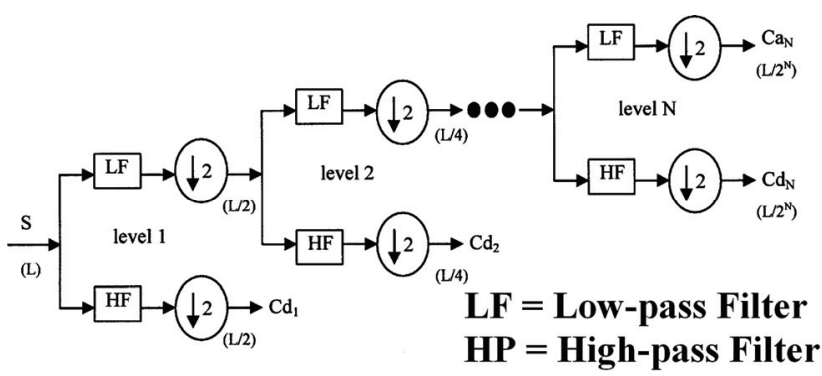

Figure 1. The tree structure of the DWT [11]; cDn is the detail .coefficient of nth decomposition level

\footnotetext{
${ }^{2}$ Quadrature Mirror Filter

${ }^{3}$ Energy definition and formulation of calculating coefficients' energy is introduced in [11].
}

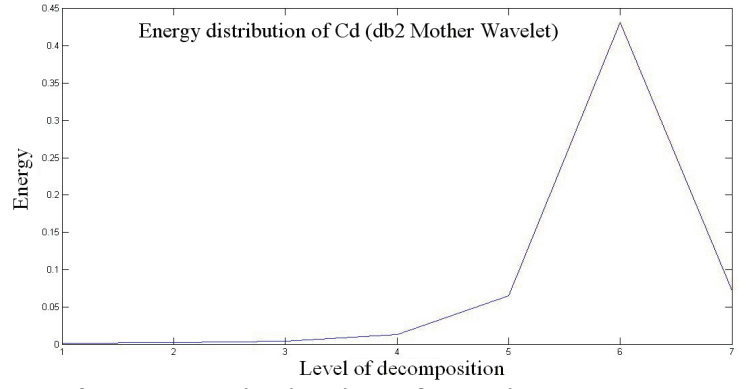

Figure 2. Energy distribution of the signal on each level, using db 2

Using mentioned method, thresholds are calculated (in fact, we train the system.). The method we calculate a threshold is called soft-thresholding and is discussed in [9]. Then decomposing is repeated and calculated thresholds are exerted to make weak samples of the coefficients zero. Weak samples are supposed to be related to noise components. Figure 3 shows the reconstructed signal using soft-thresholding in comparison with original noise-polluted PD signal.

\section{A Modification to the PD De-Noising Method}

The method introduced so far, suffers from a defect in recognizing PD sources. There are four types of signals that may reach PD-Analyzer (PDA) equipment:

1) Internal $P D$ (i.e. $P D$ pulses originating from rotating machine)

2) External PD (i.e. PD pulses originating from bus bar)

3) Internal noise (i.e. noise originating from other sources except discharges)

4) External noise (i.e. noise originating from external agents including interferences caused by communication systems or PDA itself)

The problem of the mentioned method (and generally pattern-based methods) encounter is that it cannot distinguish external PDs from internal PDs because it works based on pulse shape and is not dependant on the direction PD comes from.

Hence, a technique is needed to separate internal and external PD pulses. The method used to amend the approach works based on utilizing directional couplers [10]. Figure 4 shows the overall system measuring PD by this technique. This method (called Time-Of-Arrival) was initially used in an analogue system to separate internal and external PD [7]. But a major disadvantage of the analogue system is that one should design a system which works in frequencies upper than $50 \mathrm{MHz}$ [12] because it does not utilize any de-noising technique. Therefore, noise eliminating is done with high-pass filtering because there is no noise or interference in this system at those frequencies. 

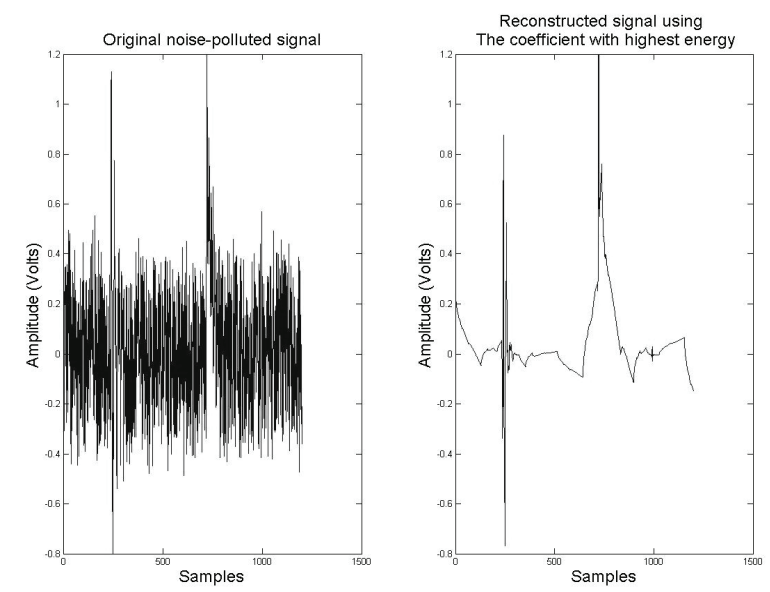

Figure 3. Noise-polluted PD pulse in comparison with the coefficient with highest energy

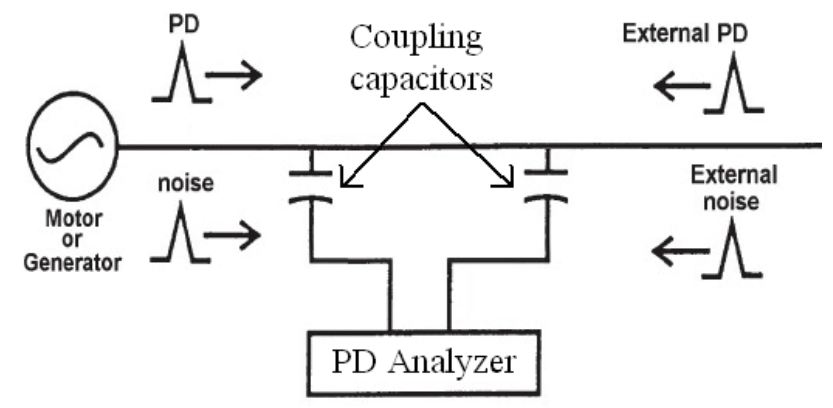

Figure 4. Different kinds of signals reaching PDA

Signals shown in Figure 4 are not generally present in every generator or motor. For example in hydro-generators there is some negligible internal noise that does not affect the measurement. Also external PD may exist or not based upon the characteristics of the bus system.

In previous sections a method to separate noise from PD was discussed. So, the only issue which is remained is to distinguish internal and external PD.

The "Time-Of-Arrival" technique helps us to separate these two signals because cable length of sensors' outputs is different so that external PD pulses arrive at PDA ports simultaneously but internal PD pulses arrive at different times [7]. By capturing the two ports of PDA in fixed sample steps using appropriate data acquisition systems $[13,14]$, we extract internal PD in two steps:

1) De-noising PD using DWT as discussed in Section 3.

2) Separating internal and external PD pulses using Time-Of-Arrival technique.

\footnotetext{
${ }^{4}$ Damped Exponentially Pulse

${ }^{5}$ Damped Oscillatory Pulse

${ }^{6} \mathrm{NEKA}$ is the most important and oldest power plant in north of Iran

${ }^{7}$ False Acceptance Rate

${ }^{8}$ False Rejection Rate
}

\section{Experimental Results}

At first step the proposed method was tested successfully on simulated data. Different kinds of noise and interference (including AM interference, sinusoid harmonies and Gaussian noise) were exerted on simulated $\mathrm{DEP}^{4}$ and DOP $^{5}$ [4] PD pulses. Then de-noising algorithm introduced before was applied to them. Figure 7 shows a sample of simulation results.

For further evaluation of the proposed method, recordings from NEKA ${ }^{6}$ power plant were made, which is equipped with $4 * 440$ MW turbo-generators. Various kinds of noise were observed at the output of coupling capacitors attached to the output of the generators. Main noise types were harmonies and Gaussian noise and the total mean SNR was about $3 \mathrm{~dB}$. Applying the proposed method to recorded signals and comparing results with observations of experts showed that the method recognizes PDs with a mean error as much as $3.2 \%$ for $\mathrm{FAR}^{7}$ and $2.9 \%$ for $\mathrm{FRR}^{8}$ (FAR happens when a noise pulse is recognized as PD and FRR happens when a PD is recognized as noise.). A sample of PD de-noising of recorded data is shown is Figure 8(a) and 8(b).

As the result of this work will be used to manufacture a PD Analyzer in NRI, major methods of de-noising PD to measure its level and frequency band had to be considered. Therefore two successful methods i.e. fuzzy logic presented in [2] and the method presented in this paper were implemented on a high technology hardware comprised of the followings:

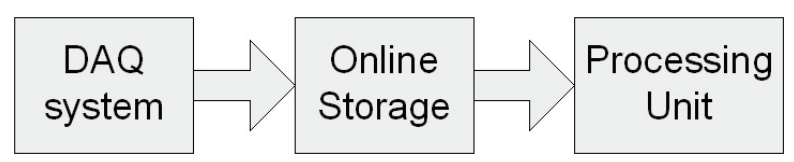

Figure 5. A simple block diagram of PD analyzer system used

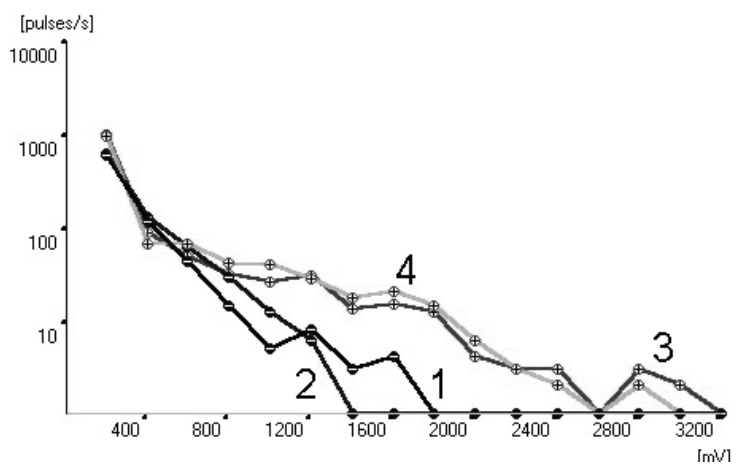

Figure 6. Pulse height diagram of $P D$ signals measured in NEKA power plant; March 2008 (diagram no.1: fuzzy method, diagram no.2: DWT method); September 2008 (diagram no.3: fuzzy method, diagram no.4: DWT method) 
1) Data acquisition system from National Instruments (NI PCI-5154) with sampling rate of $2 \mathrm{GS} / \mathrm{s}$.

2) Online storage and processing unit based on Xilinx Virtex-II FPGA.

3) Data presentation on PC in Labview software.

Figure 5 shows a simplified block diagram of the system.

Figure 6 shows the pulse height diagram of PD pulses of NEKA power plant in March (diagrams number 1 and 2) and September (diagrams number 3 and 4) 2008. Diagrams number 1 (in black color) and 3 (in red color) are related to fuzzy logic PD de-noising and diagrams number 2 (in blue color) and 4 (in green color) are the results of the method presented in this paper. Comparison shows that the method proposed in this paper yields accuracies as well as the fuzzy method, although it benefits from lower complexity.

\section{Conclusions}

DWT is a powerful method in PD de-noising of rotating machines. But it is very important that appropriate tools be used to yield enough accuracy. It was found that PD de-noising using Daubechies mother wavelets and soft-thresholding based on maximum energy of decomposition coefficients yields best results. This paper also proposed a method to obviate a defect of DWT method to separate internal and external PDs of rotating machines. The method is based on Time-Of-Arrival theory and is digitally implemented. Testing of the method showed that it can recognize PDs with a mean error as much as $3.2 \%$ for FAR and $2.9 \%$ for FRR. Finally, results of the proposed method were compared to a reference method that was using fuzzy algorithms.

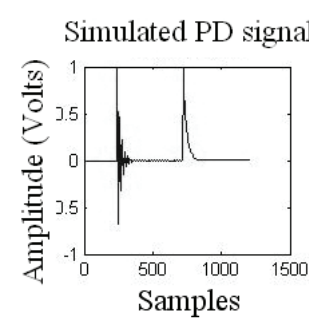

(a)

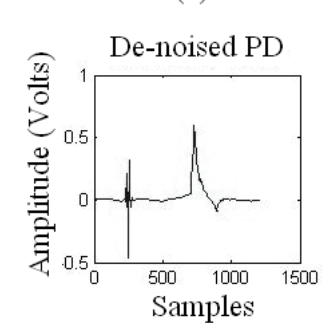

(c)

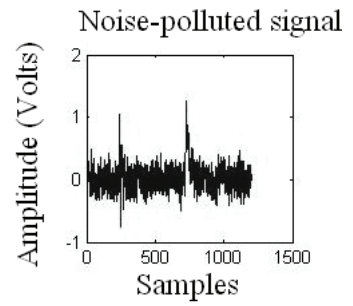

(b)
Figure 7. PD de-noising simulation results, (a) Simulated PD signal; (b) Noise-polluted PD; (c) De-noised PD using seven levels of decomposition and $\mathrm{db} 2$ mother wavelet

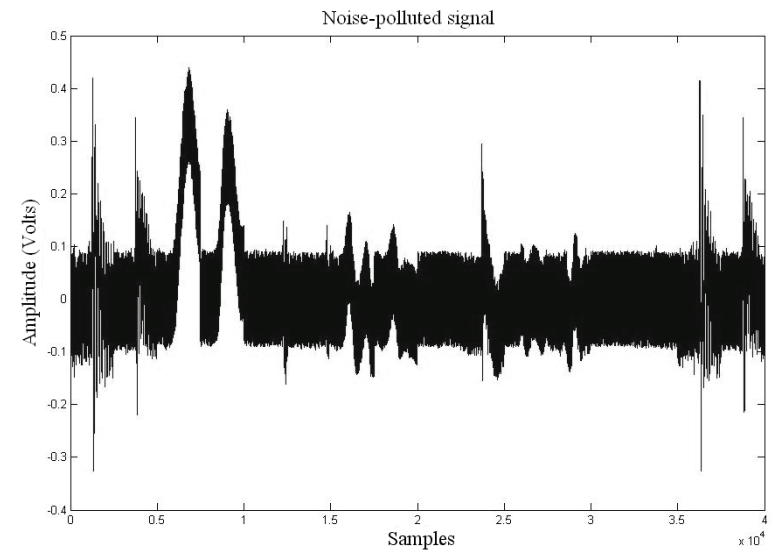

Figure 8(a). Noise-polluted signal (recorded from NEKA power plant at 27th March 2008)

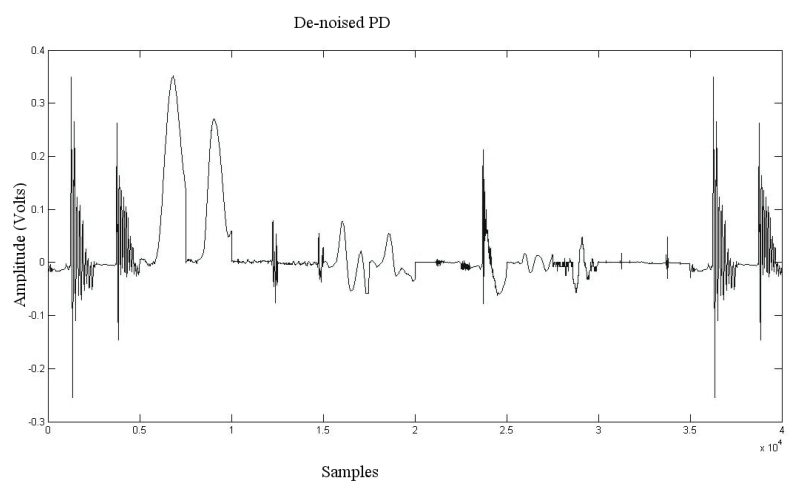

Figure 8(b). De-noised PD using proposed method

\section{REFERENCES}

[1] N. C. Sahoo, M. M. A. Salama, and R. Bartnikas, "Trends in partial discharge pattern classification: A survey," IEEE Transactions on Dielectrics and Electrical Insulation, Vol. 12, No. 2, April 2005.

[2] A. Contin, et. al., "Digital detection and fuzzy classification of partial discharge signals," IEEE Transactions on Dielectrics and Electrical Insulation, Vol. 9, No. 3, June 2002.

[3] L. Satish and B. Nazneen, "Wavelet-based denoising of partial discharge signals buried in excessive noise and interference," IEEE Transactions on Dielectrics and Electrical Insulation, Vol. 10, No. 2, April 2003

[4] H. Zhang, T. R. Blackburn, B. T. Phung, and D. Sen, “A novel wavelet transform technique for on-line partial discharge measurements Part 1: WT de-noising algorithm," IEEE Transactions on Dielectrics and Electrical Insulation, Vol. 14, No. 1; February 2007; I. S. Jacobs and C. P. Bean, "Fine particles, thin films and exchange anisotropy," in Magnetism, Vol. 3; G. T. Rado and H. Suhl, Editors, New York, Academic, pp. 271-350, 1963.

[5] X. D. Wang, et al., "Analysis of partial discharge signal using the Hilbert-Huang transform," IEEE Transactions on Power Delivery, Vol. 21, No. 3, July 2006. 
[6] S. Sriram, S. Nitin, K. M. M. Prabhu, and M. J. Bastiaans, "Signal denoising techniques for partial discharge measurements," IEEE Transactions on Dielectrics and Electrical Insulation, Vol. 12, No. 6, December 2005.

[7] G. C. Stone and V. Warren, "Objective methods to interpret partial-discharge data on rotating-machine stator windings," IEEE Transactions on Industry Applications, Vol. 42, No. 1, January/February 2006.

[8] E. Hernandez and G. Weiss, "A first course on wavelets," CRC Press, 1996.

[9] X. Ma, C. Zhou, and I. J. Kemp, "Interpretation of wavelet analysis and its application in partial discharge detection," IEEE Transactions on Dielectrics and Electrical Insulation, Vol. 9, No. 3, June 2002; J. Clerk Maxwell, "A treatise on electricity and magnetism," 3rd Edition, Vol. 2. Oxford, Clarendon, pp.68-73, 1892.

[10] IEC 60270, "High-voltage test techniques - partial discharge measurements", 3rd Edition, December 2000.
[11] X. X. Zhou, C. Zhou, and I. J. Kemp, “An improved methodology for application of wavelet transform to partial discharge measurement denoising," IEEE Transactions on Dielectrics and Electrical Insulation, Vol. 12, No. 3, June 2005; K. Elissa, "Title of paper if known," unpublished.

[12] IEEE Standard 1434-2000, IEEE trial guide to the measurement of partial discharges in rotating machinery, 2000.

[13] S. H. Wang, F. C. Lu, and Y. P. Liu, "High-speed data acquisition system for partial discharge on-line monitoring in transformer," Proceedings of the 14th International Symposium on High Voltage Engineering, Tsinghua University, August 2005.

[14] J. Borghetto, "Partial discharge inference by an advanced system: Analysis of online measurements performed on hydro-generator," IEEE Transactions on Energy Conversion, Vol. 19, No. 2, June 2004. 